\title{
Dependability of the Users in Cloud Environment Using load Balancing and Integrity of Data
}

\author{
Sandhiya R, D.Radhika., G.Sasikala
}

\begin{abstract}
A distributed computing is one of the most amazing administration offered by means of web which makes a stage to store and recover information Since client's close to home information is being put away in a decoded arrangement on a remote machine worked by outsider merchants who give different administrations, the effect of client's personality and unapproved access or revelation of records are extremely high.In this proposed system is using Dependability of the Users,Privacy and Integrity of Data (DPI) algorithms using improve the Execution assessment of Cloud Computing foundations is required to anticipate and measure the money saving advantage of a technique portfolio and the relating Quality of Service (QoS) experienced by clients. Along these lines, concentrating on Load adjusting in the distributed computing condition importantly affects the presentation. This paper deals with multi target asset provisioning plan for taking care of different errand classes for different outstanding task at hand office. In this proposal, project is using a Best Partition Searching for distributing a file system to another cloud environment. This methodology gives a security regarding client verification for "approval" to enter the system which is made by means of Image Sequencing secret key to demonstrate that the personality is unique client, RSA calculation to encode the information to give information respectability, a profoundly parallel conveyed information the executives administration that empowers to peruse/compose and add gigantic informational collections that are divided and circulated at a huge scale. Subsequently this methodology gives a general security to the customer's close to home information and the issue of simultaneousness, volume of information can be settled with these procedures.
\end{abstract}

KEYWORDS: - Integrity of Data, Dependability of the Users, Quality of Service, Load balancing

\section{INTRODUCTION}

Today, this propose have a capacity to use versatile, circulated registering conditions inside the bounds of the Internet, a training known as distributed computing. In this new universe of figuring, clients are all around required to acknowledge the basic reason of trust. Inside the distributed computing world, the virtual condition lets clients' entrance figuring power that surpasses that contained inside their own physical universes. Distributed computing is the way toward giving PC offices by means of web. Furthermore, it's given us better and effective approach to get to data in auspicious

Revised Manuscript Received on August 14, 2019.

Sandhiya R, PG scholar, Department of CSE, Vivekanandha College of Engineering for women (Autonomous), Tiruchengode, India. (sandhiyageniune@gmail.com)

D.Radhika., M.E., Assistant Professor, Department of CSE, Vivekanandha College of Engineering for women (Autonomous), Tiruchengode, India. (radhikadevacse@gmail.com) Vivekanandha College of Engineering for women (Autonomous), Tiruchengode, India. (sasimecse2013@gmail.com)
G.Sasikala. M.E., Assistant Professor, Department of CSE,

way and furthermore expands capacity of limit with respect to client in.

Distributed computing empowers another plan of action that supports on-request, pay for-use, and economies-ofscale IT benefits over the Internet. The Internet cloud fills in as an administration industrial facility worked around virtualized information centers. 1 Cloud stages are progressively worked through virtualization with provisioned equipment, programming, systems, and datasets. The thought is to move work area registering to an administration situated stage utilizing virtual server bunches at server farms. Nonetheless, an absence of trust between cloud clients and suppliers has ruined the general acknowledgment of mists as re-appropriated figuring administrations. To advance multi-vitality, this propose must structure the cloud environment to be secure, rust commendable, and trustworthy. In actuality, trust is a social issue, not a simply specialized issue. In any case, we accept that innovation can improve trust, equity, notoriety, believability, and affirmation in Internet applications. To expand the appropriation of Web and cloud administrations, cloud specialist organizations (CSPs) should initially build up trust and security to reduce the stresses of countless clients.

Cloud clients are on edge about whether server farm proprietors will abuse the framework by self-assertively utilizing private datasets or discharging touchy information to a third party Without consent. Cloud security is sent to give loaded with insurance between information proprietor and specialist co-op. To address these issues, this propose a notoriety based trust-the board plan enlarged with information shading and programming watermarking.

The Cloud Security Alliance 5 has recognized a couple of basic issues for believed distributed computing, and a few ongoing works examine general issues on cloud security and protection. Open and private mists request various degrees of security requirement. This propose can recognize among various administration level understandings (SLAs) by their variable level of shared duty between cloud suppliers and clients. Basic security issues incorporate information honesty, client privacy, and trust among suppliers, singular clients, and client gatherings. The three most well known cloud administration models have fluctuating security requests; the foundation as-an administration (IaaS) model sits at the deepest usage layer, which is stretched out to shape the stage as-an administration (PaaS) layer by

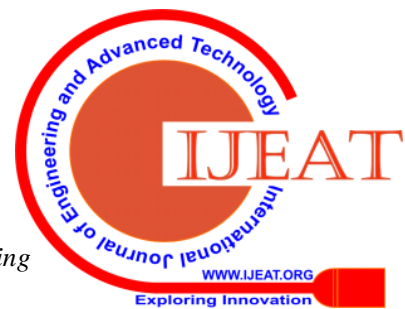



OF DATA

including OS and middleware support. PaaS further stretches out to the product as-an administration (SaaS) model by making applications on information, substance, and metadata utilizing uncommon APIs. This infers SaaS requests all insurance capacities at all levels. At the other extraordinary, IaaS requests insurance for the most part at the systems administration, confided in processing, and figure stockpiling levels, while PaaS typifies the IaaS support in addition to extra assurance at the asset the executives level.

\section{Virtual Trust Difficulty in Cloud Services:}

The cloud security gathering has distinguished a couple of fundamental issues for believed distributed computing, and a few late works talk about general issues on cloud security and protection. Open and private mists request various degrees of security authorization. This propose can separate among various administration level understandings (SLAs) by their variable level of shared obligation of Security concerns which contains information honesty, client secrecy, and trust among suppliers, singular clients, what's more, client gatherings. The three security requests ought to be changed from the three cloud administration models that are depicted in beneath the foundation as-an administration model sits at the deepest usage layer, which is extended to shape the stage as-an administration (PaaS) layer by including OS and middleware support. Pass further reaches out to the product as-an administration ( $\mathrm{SaaS}$ ) model by making applications on information, content, and metainformation utilizing uncommon A PIs. This infers SaaS requests all security capacities at all levels.

\section{Verifying transportation as an administration}

The Iaas model is attempts to figure systems administration and information stockpiling, different assets in a virtualized situation. Amazon's Elastic Compute cloud is one of genuine case of Iaas, at the cloud foundation level, CSP can execute organize security with interruption identification frameworks, firewalls, antivirus programs, disseminated refusal of-administration doubt, etc. Verifying approach as an administration. Cloud stages worked in Iaas with framework joining and virtualization center product. Also, these stages can be utilized to clients for executing client assembled programming applications onto the cloud framework utilizing supplier bolstered programming dialects and programming devices like Java, Python and Dot net.

\section{Cloud Suppliers and Reported Services}

Equipment and programming highlights are available in cloud security. Three primary security prerequisites is utilized in distributed computing requests, for example, classification, respectability and accessibility. Report is the way toward keeping up client conveyed subtleties on database. Furthermore, these subtleties are seen distinctly by a validated client in cloud situations

Information unwavering quality and privacy Protection nformation trustworthiness is the capacity of cloud supplier guard information from unapproved individual or programmers. Classification is basically the manner in which the cloud supplier safeguards that the information is verified from unapproved get to. The measures that the cloud supplier uses to protect that this objective is met incorporate physical disconnection and cryptology. Distributed computing is an open system, which brings a lot of entangled difficulties for the supplier to create disconnection for the client. Physical seclusion is cultivated by utilizing virtual neighborhood and center boxes. The second technique the supplier uses is cryptology, which basically scrambles the information before it is set into the cloud. These two strategies are standard estimates that are utilized to verify information in the cloud.

Numerous devices are accessible for developing cloud applications on enormous datasets and it's given by cloud programming condition to wanted clients. We should following highlights are available in security and protection, for example, Fine-grained access control to preserve information respectability and prevent interlopers or programmers a strategy to prevent ISPs or CSPs from assaulting client protection. CSPs that battle against spyware and web bug. We can improve a portion of these highlights with cloud notoriety frameworks and progressively productive personality the board frameworks a few highlights are Cloud asset can get to security convention like http and secure attachment layer. Fine grained access control to ensure information respectability and information aggressor.

Believed Cloud Computing over Data Centers Security mindful cloud design and this used to recognize the assurance components required. Gatecrasher discovery activity is ought to be executed by utilizing these models.

Cloud insurance Infrastructure Trusted and trustworthy cloud design ensures system assault by propelling confided in operational areas for various cloud applications. The challenges in security understanding that CSPs ensure all information jog servers and capacity zones. Our design shields MACHINE checking from programming based assaults and upholder information and data from burglary, extortion and common disappointments. It gives solid validation and approved access to delicate information and on-request benefits. This propose had a few structure targets for a trusted and reliable cloud when making our engineering.

\section{RELATED WORKS}

Burden adjusting is a proficient piece of distributed computing condition which affirms that all strategies work comparable measure of work specifically timespan. The various kinds of calculations for burden adjusting over cloud condition have been executed with the principle objective to create cloud assets open to the end clients with simple and availability. The primary burden adjusting issue is the run time over-burden owed to the difference in burden information among CPUs, determination of procedures for basic leadership and moves the activity from processor to processor. The proposed methodology investigates the conditions and partitions the heap adjusting approach in different layers [1].Recent promising advancements in quantum registering appear to be perfect for playing out this overhead work. As a stage towards this objective, this paper proposes a host asset utilization expectation approach, in light of a complex- 
esteemed neural system. The calculation can be additionally altered later on to be material to quantum processing situations. A proof-of-idea is assessed on genuine burden follows from a framework. The calculation is thought about against some present cutting edge have load forecast calculations to show its exactness [2].

Distributed computing is turning into a feasible processing answer for administrations arranged registering. A few open-source cloud arrangements are accessible to these backings. Open-source programming stacks offer a tremendous measure of adaptability without colossal permitting expenses. Accordingly, open source programming are broadly utilized for structuring cloud, and private mists are being fabricated progressively in the open source way. Various commitments have been made by the open-source network identified with private-IaaS-cloud. Open Nebula - a cloud stage is one of the mainstream private cloud the board programming [3]. In this mathode the time arrangement of day by day most extreme burden is parceled into two sections, chronicled dataset and current propensity dataset, in reverse cloud calculation is connected to the two datasets to frame the verifiable cloud and the present cloud, and the comparing principle sets are mined. At that point the authentic cloud and current cloud is coordinated to made prescient cloud through blended cloud. At long last, through cloud thinking, the figure result can be acquired. This prescient technique adequately coordinates semi periodical consistency and current propensity of timearrangement information, and has a basic processing model. The contextual investigation demonstrates that the proposed strategy is precise and useful [4].

These days treatment of enormous information move among a large number of interconnected servers assumes a fundamental job on distributed computing condition. Huge information is only accumulation of social information, unstructured information (comprehensible organization), and semi-organized information, gushing information, for example, machines, sensors, Web applications, and internet based life. In existing framework this idea upgrades by fixing some ideal to defeat the bottlenecks happens while information move on logical cloud application. The parameters are pipelining, parallelism, and simultaneousness [5].Researchers utilized numerous approaches (for example Insect province, Honey Bee, Genetic, Static, and Dynamic) yet need improvement. Virtual Machines (VMs) movement framework assumes the imperative job in burden the executives. Our concentration in this examination is amplifying the use of VMs CPU limit. Proposed research approach depends on Dynamic Weighted Live Migration (DWLM) to oversee load awkwardness issue. The proposed system results contrasted and Migration time, Scalability, Throughput and Availability factor from Equally Spread Current Execution burden adjusting calculation (ESCEL) and Push Pull calculation. This paper additionally centers around others burden adjusting methodologies and future research scope in Load the board component [6].

Confronting load irregularity issue; that is, the record lumps are not doling out effectively among every one of the hubs. For that reason, the heap rebalancing strategy comes into picture, load rebalancing has the more productivity that makes framework progressively effective. For burden lopsidedness we utilize a safe burden rebalancing calculation that converges with the MD5 with DES encryption calculation. The outcome shows play out the framework as far as security parameters message overhead and the development cost for our proposed plan. For this work we are executing in disseminated document arrangement of the EC2 [7]. Scheduling of computational load and actual processing is an important problem to be considered from the perspectives of time and consumed energy for execution in the scale of data centers. In this paper, time-series analysis of the arrivals of the workloads have been done by applying auto regression (AR), moving average (MA), auto regression and moving average (ARMA), and Holt-Winters approaches. Performances of the four methods was evaluated and compared for Google workload $\operatorname{logs}$ that is publicly available in the Internet [8].

Distributed computing is a developing processing worldview in which shared assets are given by the client demand at explicit time. Burden adjusting is the way toward dispersing outstanding task at hand among different hubs of the figuring framework. The heap can be CPU load, memory limit, or system load. A proficient burden adjusting keeps away from a circumstance where a portion of the hubs are vigorously stacked while different hubs are inert or doing almost no work. At the point when Virtual Machine (VM) is over-burden with numerous undertakings, these errands are expelled and relocated to the under stacked VMs of the equivalent or distinctive datacenter [9]. The fundamental goal of this paper was to propose cloud supporter structure to help cloud for preparing numerous assignments. Due to various errands on cloud server, it works increasingly slow gets fizzled. In this circumstance dispersing or planning of assignments on the conveyed registering framework is the main answer for decrease remaining burden on server. Processing capacity of advanced mobile phones CPU can contend with the CPU of the PCs. Advanced mobile phones are vitality productive and financially savvy option in contrast to running certain errands of conventional servers. Conveying undertakings for calculations must use every one of the assets similarly, no asset ought to be under or over use this issue prompts center around the heap adjusting method to help the cloud for handling assignments [10].

Distributed computing is offering on interest benefits by giving virtualize and capacity condition. Because of the sharing of assets in cloud, there is an opportunity of asset wastage which makes requirement for burden adjusting. To address this issue, this work proposes a versatile arrangement which improves the presentation of the circulated frameworks and gives adaptability wanted in web based registering conditions. The proposed calculation has been actualized and contrasted and the aftereffects of existing components and consequences of our proposed system has been discovered promising [11]. In Cloud based Storage each server bunches totally handles a specific sort of data administration and gets the customer's solicitation powerfully in various time steps. It is an examination challenge to structure a productive burden adjusting 
calculation which can dole out the mixed media message employments with least cost between server groups and customers while not over-burdening the server bunches. Varying from past works, this paper considers a progressively commonsense powerful benefits situation in which every server bunches handles just a specific sort of sight and sound assignment and every customer demand diverse kind of media administrations at various time. Such situation can be dealt with as a whole number straight programming issue which is computationally in attainable by and large. In this paper an endeavor to take care of the issue by a productive GA in foreigner plan Simulation results show that the proposed hereditary calculation can effectively adapt to dynamic multi-administration burden adjusting in CMS [12].

Various colossal cloud associations, alike as Amazon, Yahoo, Google, offer many cloud-benefits and have numerous clients. Distributed computing is an Internetbased registering approach, where the product, assets and the applications are shared between many-to-many figuring gadgets. Cloud Load-adjusting (CLB) takes the abundance of the cloud's versatility and the physically to meet rerouted outstanding burden and to improve generally speaking accessibility. In the expansion of assignments products and traffic conveyance, CLB innovation gives wellness checks to the cloud applications. We utilized GA way to deal with taking care of the LB in distributed computing. Our proposed work is more suitable than the present procedures work, as we executed the cloudlets in less time and playing out the heap adjusting in greater benefit [13].

Distributed computing is one of the quickly developing fields in superior processing. The interest for distributed computing administrations is expanding because of its accessibility anyplace whenever. As the clients for distributed computing administration increments quickly, the heap on individual hubs increments. This circumstance triggers the requirement for burden adjusting. Burden adjusting is a technique by which the client solicitations are similarly circulated among the accessible virtual machines in the server farm to accomplish most extreme throughput, handling time and reaction time. In our work, we are fusing Weighted Round Robin calculation in Honeybee calculation so as to accomplish least server farm preparing time and reaction time [14]. Clients can utilize these assets and administrations as they need on compensation per use idea. In distributed computing engineering burden adjusting is a significant issue. There are numerous calculations for burden adjusting in distributed computing. All calculations work various ways. We proposed an Improved Max-Min Ant state Algorithm. Improved Max-Min utilized the idea of unique Max-Min. Improved Max-Min depends on the execution time not on finish time as a determination premise. The principle rationale of our work is to adjust the complete heap of cloud framework. We attempt to limiting the complete makespan. We recreated results utilizing the CloudSim toolbox. Results demonstrate the correlation between improved max min and new half and half improved Max-Min subterranean insect approach. It primarily centers around all out handling time and preparing cost [15].

\section{METHODS AND IMPLEMENTATIONS}

Current accessible cloud sellers give gigantic measures of extra room and figuring assets get to the client information from Cloud stockpiling protection and security issues are significant worry that should be settled we should utilize new strategy for cloud capacity security upgrade. By actualizing Cloud stockpiling numerous business related security issues and issues and dangers will be settled. To comprehend the security issues related with cloudStorage. To give amazing administrations to the users. To give high information security in cloud basedEnvironment utilizing steganography, encryption andDecryption..To minimizing the data uploading and downloadingTime on cloud storage. In this proposed system is using Dependability of the Users,Privacy and Integrity of Data (DPI)algorithm depend upon In cloud based environment there are many security issues such as authentication, integrity, privacy, virtualization, confidentiality, large amount data processing, scalability, access control etc. Traditional security approaches are no longer suitable for data and application in cloud.

Cloud Computing have scalable and location independence features so application and data stored in cloud have no fixed limitations. In security breaches it is quite difficult to resolve a particular node in which threats occurred. Due to the openness of cloud environment data may be accessed by unauthorized users. In cloud the issue of verifying correctness of cloud data storage becomes more challenging. Cloud computing poses several security threats due to number of reasons. Data Breaches is also major security concern in cloud storage. User stored large data sets in the cloud so there is a chance that malicious user may entered in the cloud storage system. There is high possibility of attack and threats. In cloud storage data integrity must be kept effectively to avoid data loss. In cloud storage data is stored over the remote server so it is necessary to preserve confidentiality. Security policies should be followed strictly

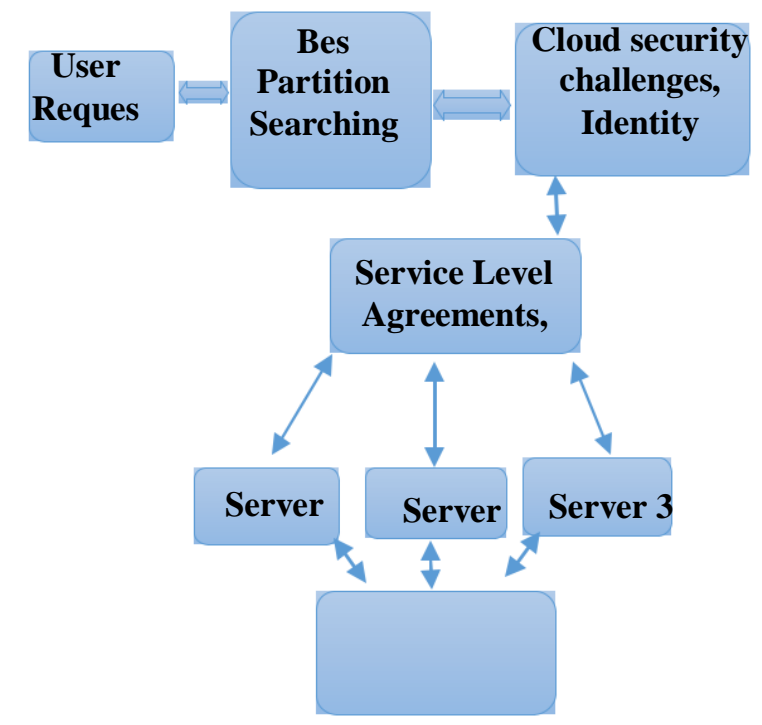

Fig: Dependability of the Users,Privacy and Integrity 
In cloud the issue of confirming accuracy of cloud information stockpiling turns out to be all the more testing. Distributed computing represents a few security dangers because of number of reasons. Information Breaches is likewise real security worry in distributed storage. Client put away huge informational collections in the cloud so quite possibly noxious client may entered in the distributed storage framework. There is high probability of assault and dangers. In distributed storage information trustworthiness must be kept viably to stay away from information misfortune. In distributed storage information is put away over the remote server so it is important to safeguard secrecy. Security arrangements ought to be pursued carefully.

\section{Personality the board and access control}

In enlistment module client will enlist themselves by client name, secret key, contact number and email id. Client produce irregular check code by utilizing Random recurrence. Client get the confirmation code on his email address after that client check the code and divert to landing page. On the off chance that confirmation code field stay clear or client entered wrong check code, at that point divert to login page. Login is the strategy by which individual gain admittance to the information by recognizing and validating through the certifications given by the client. Client has logout when access is never again required.

\section{Administration Level Agreements}

In this recommended framework record is dissipated at three areas. Initial one is at our application and next two where second and third documents are put away. We created setting page that will be utilized for transferring

\section{Cloud database}

what's more, downloading document from table. Addition FTP subtleties into table. As such splitted documents get spared to various cloud server. We made a strategy where client can share documents to different clients, for that we have structured a page wherein client can essentially enter the id of individual whom to move the records and document gets transferred to cloud server and name of the documents get spared to SQL server table. The collector will get a warning that someone has imparted a document to you. In the event that client taps on the download catch all the splitted documents get blended and spared to collector neighborhood framework. Presently the collector gathering gets the encoded and packed document the time client needs to unscramble and decompressed the got record.

\section{Information Privacy and Integrity}

In Suggested framework, we are parting the information record, picture document or video in various parts with some augmentation (.part in our case).After parting we put away splatted record in our nearby framework with expansion .part. In this proposed framework, scramble every single splitted document which is of .part augmentation with open key so it can't be effectively discernible by any unapproved access or programmer.

Encryption system like DES, AES, and RSA is created before putting away it on cloud. In this proposed framework, splitted documents get compacted with GZIPSTREAM calculation so the size of splitted records gets diminished, and it can without much of a stretch be moved to cloud server. Zip depends on the collapse calculation.

\section{Super Key Assessment Algorithm Step 1:}

Cipher(no of byte in [6*TNb] and No of byte out $\left[\mathrm{TNb}^{*}(\mathrm{TNr}+1)\right]$,

Start

Byteformal [6, TNb], Formal $=$ in

\author{
Step 2: \\ If $(\mathrm{TNr}=$ in $\mathrm{TNb})$ \\ \{ \\ AddRoundKey(formal, w[0, Nb-1]) \\ For pattern Cipher key= 1 step 1 to $\mathrm{TNr}-1$ Split Sub \\ Bytes (formal) Data Rows Shift (formal) \\ Mix data columns and rows (formal) \\ Addpattern CipherKey(formal, \\ W [pattern Cipher Key ${ }^{*} \mathrm{TNb}$, (pattern Cipher Key \\ $\left.+1)^{*} \mathrm{TNb}-1\right]$ )
}

End for

Addpattern Cipher Key (formal, w
$[\mathrm{TNr} * \mathrm{Nb}$,
$\left.\left.(\mathrm{TNr}+1)^{*} \mathrm{TNb}-1\right]\right)$
\}
Else
\{
Out = formal
\}

Step 3:

End

The figure instant message contains all the data of the plaintext message, yet isn't in a configuration Readable by a human or PC without the best possible component to decode it; it ought to look like arbitrary drivel to those not expected to peruse it. The scrambling system is fluctuated relying upon the key which changes the nitty gritty activity of the calculation. Without the key, the figure can't be utilized to encode or unscramble.

\section{Data Distribution}

Data distribution is a critical task distribution should maximize transfer rates and minimize information redundancy. VMs algorithm to reduce the burden in virtual machine. Set of heuristics that prevent burden in the system effectively while saving energy used. The second component is a central data repository which has the files to be deployed onto all the VMs.

\section{Algorithm JSQ ()}

\{

Scheduler initializes the VM allocation table. While (there is a task received by JSQ scheduler) \{ 
$\mathrm{VM}$

Scheduler forwards the task towards that

Whose queue length is smallest \& update VM allocation table.

If (any virtual machine completes the task) Then

\{

Update the VM allocation table Accordingly.

\}

\}

\section{Steps of proposed algorithm}

Stage 1: Through scheduler table pick the scheduler whose inactive line length is biggest, allocate the undertaking to sit virtual machine through the chose scheduler and update the scheduler table as needs be.

Stage 2: If inactive line of all scheduler is unfilled then Scheduler get to the VM table and select that virtual machine whose line length is briefest and relegate the undertaking to that virtual machine.

Stage 3: On finishing of errand, VM update the VM table. On the off chance that any VM gets inert, at that point it adds itself to the inactive line of that scheduler whose line length is most limited. Scheduler refreshes the scheduler table in like manner.

Key Expansions-Round keys are gotten from the figure key utilizing Rijndael's key calendar. AES requires a different 128-piece round key square for each round in addition to one more.

\section{Particle Swarm Optimization}

The predefined number of VMs are made and began inside the chose cloud supplier. This capacity plays out the genuine organization of the virtual framework, establishment, and arrangement of the product introduced in the VMs in our particular case it is our VMs and the friends for disseminating the parceled document. Programmed Deployment Layer utilizing the arrangement parameters taken from the client. In this situation one of the VMs is haphazardly picked to be the ace and the others become captives of the application.

\section{RESULT AND DISCUSSION}

\section{Analysis of time complexity}

The time complexity parameter has been calculated by using database and the request queries are input of the listed algorithm based on the number of users and the execution time of the individual algorithms are represented below: Time represented by $\mathrm{T}$, Data analytics by DA, Trust Accuracy represented by TA

\section{$\mathrm{T}=\frac{+}{\text { No database access }+ \text { no of user }}$}

The figure given below shows the Time complexity by different comparisons as follows

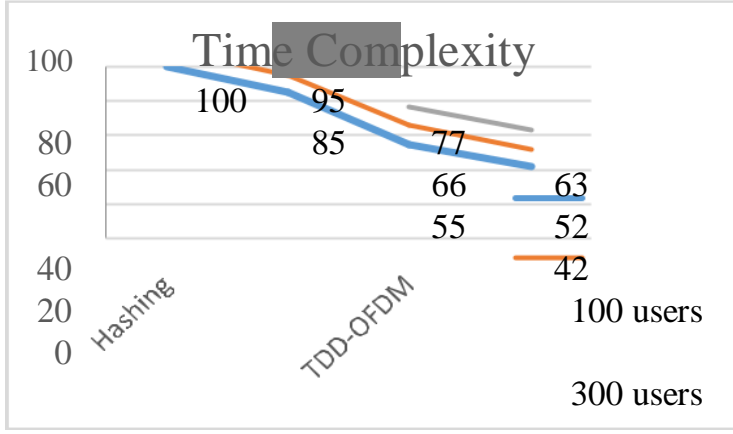

\section{Table 6.1.2 Processed data, UN Processed data and Efficiency of packet processing}

Table 4.4 represents the throughput ratio of the different algorithmsDPI $73 \%$, Cipher Key $68 \%$, SKA $57 \%$ and Hashing $42 \%$ and the overall the proposed DPI technique have the high throughput ratio as compared with other techniques.

The resultant algorithm has been implemented and evaluated for its performance using the dataset being considered with previous clarification. The method has produced efficient results in all the factors considered.

\section{Analysis of Integrity to security provision}

The figure given below shows the false ratio accuracy by different comparisons as follows

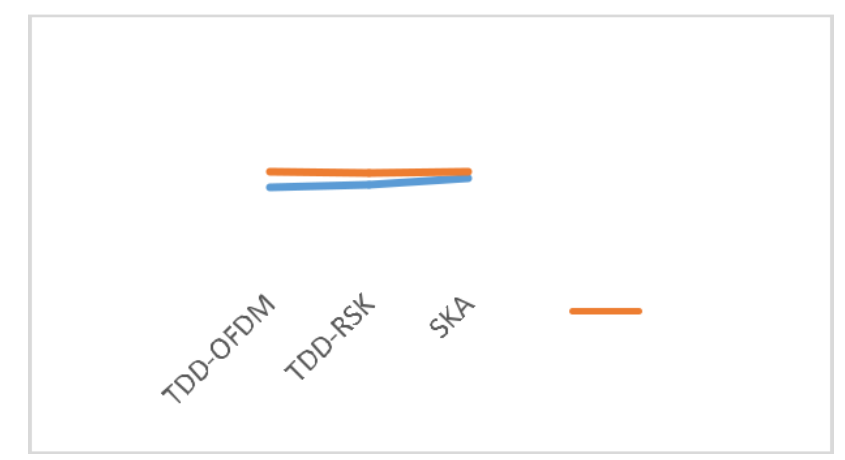

Figure 6.1.2: Comparison of time complexity

Figure 4.10, shows the comparative result on time complexity produced by various methods and shows clearly that the proposed SKA has produced less time complexity.

\begin{tabular}{|c|c|c|c|c|}
\hline Users & $\begin{array}{c}\text { Hashin } \\
\text { g }\end{array}$ & $\begin{array}{l}\text { Ciphe } \\
\text { r key }\end{array}$ & $\begin{array}{r}\text { SK } \\
\text { A }\end{array}$ & DPI \\
\hline $\begin{array}{l}100 \\
\text { Users }\end{array}$ & 100 & 85 & 55 & 42 \\
\hline $\begin{array}{c}300 \\
\text { Users }\end{array}$ & 110 & 95 & 66 & 52 \\
\hline 500 & 125 & 105 & 77 & 63 \\
\hline
\end{tabular}


Integrity to security provision

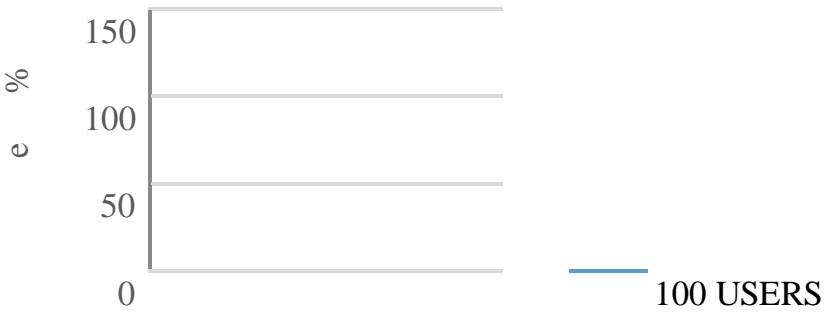

Figure 6.1.4 various dissimilar in Integrity to security provision

Figure 4.2.9 shows the comparative result on intent security produced by different methods. The proposed TDDRSK method has produced high performance in integrity to security dissimilar than other methods. They represent the Integrity in security performance of the algorithms, SKA algorithm 90\% TDD-OFDM 72\% and TDD-RSK technique $75 \%$ and the overall the proposed SKA technique have the high Integrity Security as compared with other techniques.

\begin{tabular}{|c|c|c|c|}
\hline USERS & $\begin{array}{l}\text { TDD- } \\
\text { OFDM }\end{array}$ & $\begin{array}{l}\text { TDD- } \\
\text { RSK }\end{array}$ & SKA \\
\hline 300 Users & 92 & 95 & 103 \\
\hline 500 Users & 112 & 110 & 112 \\
\hline
\end{tabular}

Table 6.1.4 Processed Data, UN Processed Data and Efficiency of packet processing.

\section{CONCLUSION:}

Distributed storage protection and security issues are significant worry that should be settled we should utilize new technique for cloud capacity security improvement. By actualizing Cloud stockpiling numerous business related security issues and issues and dangers will be settled. By execution of this proposed work we can expanded distributed storage security utilizing encryption, decoding, pressure, sharing procedure. The compressive detecting strategy is supplanted by the DPI calculation. Utilization of DPI calculation gives us a compacted information's with high caliber. Further the parting of information into pieces then the arrangement of lumps spared into a metadata record and scrambling it utilizing AES gives further security to the information. To open secure record, client must need safely their classified document away in secure way or client can safely move their private documents over the system. By this key, all information will be in scrambled way. This methodology is very helpful on the grounds that it empowers client to repel the unapproved individual with the end goal that he can't almost certainly peruse client records. A Particle Swarm Optimization approach thinks about different credits to plan the remaining burdens. This fake insightful play out a programmed work planning. That straightforwardly relocates just the working arrangement of an inactive $\mathrm{VM}$ and bolster green registering by streamlining the quantity of servers being used. We utilize the most extreme priority calculation to lessen the weight in virtual machine.

\section{REFERENCES:}

1. Enhanced load balancer with multi-layer processing architecture for heavy load over cloud network Navdeep Singh Randhawa 19-21 Oct. 2017.

2. Towards Quantum Computing Algorithms for Datacenter Workload Predictions Kashifuddin Qazi , Igor Aizenberg 2-7 July 2018.

3. An experimental study of load balancing of OpenNebula open-source cloud computing platform A B M Moniruzzaman ; KawserWazedNafi ; Syed Akther Hossain 23-24 May 2014

4. A Novel Cloud Theory Based Time-series Predictive Method for Middle-term Electric Load Forecasting X.M. Yang, J.S. Yuan, H.N. Mao, J.Y. Yuan 4-6 Oct. 2006

5. Big data transfers through dynamic and load balanced flow on cloud networks C. Jayashri P. Abitha, S. Subburaj 27-28 Feb. 2017

6. Dynamic weighted virtual machine live migration mechanism to manages load balancing in cloud computing Pradeep Kumar Tiwari ,Sandeep Joshi 15-17 Dec. 2016

7. New approach for load rebalancer, scheduler in big data with security mechanism in cloud environment Priyanka A. Dhande, A. J. Kadam 2-3 Dec. 2016

8. Cloud work load prediction through different models based on time-series" İlksencaglar, D. TurgayAltıla 5-8 Oct. 2017

9. Load Balancing of Tasks in Cloud Computing Environment Based on Bee Colony Algorithm K R RemeshBabu ,Amaya Anna Joy, Philip Samuel 2-4 Sept. 2015

10. A Load Balancing Framework in Cloud to Support Task Processing Using Smartphone with CWC Anantkumar Vikas Salame, Prof. Pradnya V. Kulkarni 6-8 April 2018.

11. Adaptive Framework for Load Balancing to Improve the Performance of Cloud Manisha Malhotra, Aarti Singh 13-14 Feb. 2015

12. Dynamic load balancing in cloud based multimedia system with genetic algorithm K V Kavitha, Vinza V Suthan 26-27 Aug. 2016

13. Load balancing in cloud computing using genetic algorithm Monika Lagwal, Neha Bhardwaj 15-16 June 2017

14. Incorporating weighted round robin in honeybee algorithm for enhanced load balancing in cloud environment Nithin K. C. Das, Melvin S. George, P. Jaya 6-8 April 2017

15. Dynamic combination of improved max-min and ant colony algorithm for load balancing in cloud system Navtej Singh Ghumman, Rajwinder Kaur 13-15 July 2015 